\title{
Cost-benefit analysis of tranexamic acid and blood transfusion in elective lumbar spine surgery for degenerative pathologies
}

\author{
Jeff Ehresman, BS, ${ }^{1}$ Zach Pennington, BS, ${ }^{1}$ Andrew Schilling, BA, ${ }^{1}$ Ravi Medikonda, BA, ${ }^{1}$ \\ Sakibul Huq, BS, ${ }^{1}$ Kevin R. Merkel, ${ }^{2}$ A. Karim Ahmed, BS, ${ }^{1}$ Ethan Cottrill, MS, ${ }^{1}$ \\ Daniel Lubelski, MD, ${ }^{1}$ Erick M. Westbroek, MD, ${ }^{1}$ Salia Farrokh, PharmD, ${ }^{3}$ Steven M. Frank, MD, ${ }^{2}$ \\ and Daniel M. Sciubba, MD'1
}

Departments of ${ }^{1}$ Neurosurgery and ${ }^{2}$ Anesthesiology, Johns Hopkins School of Medicine; and ${ }^{3}$ Department of Pharmacy, Division of Critical Care and Surgery Pharmacy, Johns Hopkins Hospital, Baltimore, Maryland

\begin{abstract}
OBJECTIVE Blood transfusions are given to approximately one-fifth of patients undergoing elective lumbar spine surgery, and previous studies have shown that transfusions are accompanied by increased complications and additional costs. One method for decreasing transfusions is administration of tranexamic acid (TXA). The authors sought to evaluate whether the cost of TXA is offset by the decrease in blood utilization in lumbar spine surgery patients.

METHODS The authors retrospectively reviewed patients who underwent elective lumbar or thoracolumbar surgery for degenerative conditions at a tertiary care center between 2016 and 2018. Patients who received intraoperative TXA (TXA patients) were matched with patients who did not receive TXA (non-TXA patients) by age, sex, BMI, ASA (American Society of Anesthesiologists) physical status class, and surgical invasiveness score. Primary endpoints were intraoperative blood loss, number of packed red blood cell (PRBC) units transfused, and total hemostasis costs, defined as the sum of TXA costs and blood transfusion costs throughout the hospital stay. A subanalysis was then performed by substratifying both cohorts into short-length (1-4 levels) and long-length (5-8 levels) spinal constructs.
\end{abstract}

RESULTS Of the 1353 patients who met inclusion criteria, 68 TXA patients were matched to 68 non-TXA patients. Patients in the TXA group had significantly decreased mean intraoperative blood loss (1039 vs $1437 \mathrm{~mL}, p=0.01$ ). There were no differences between the patient groups in the total costs of blood transfusion and TXA $(p=0.5)$. When the 2 patient groups were substratified by length of construct, the long-length construct group showed a significant net cost savings of $\$ 328.69$ per patient in the TXA group ( $p=0.027$ ). This result was attributable to the finding that patients undergoing long-length construct surgeries who were given TXA received a lower amount of PRBC units throughout their hospital stay (2.4 vs 4.0, $p=0.007$ ).

CONCLUSIONS TXA use was associated with decreased intraoperative blood loss and significant reductions in total hemostasis costs for patients undergoing surgery on more than 4 levels. Furthermore, the use of TXA in patients who received short constructs led to no additional net costs. With the increasing emphasis put on value-based care interventions, use of TXA may represent one mechanism for decreasing total care costs, particularly in the cases of larger spine constructs.

https://thejns.org/doi/abs/10.3171/2020.1.SPINE191464

KEYWORDS lumbar spine surgery; blood loss; tranexamic acid; transfusion; cost

$\mathrm{O}$ F patients who undergo elective lumbar spine surgery annually in the United States, ${ }^{1}$ approximately $17 \%$ of patients ${ }^{2}$ will require allogeneic blood transfusion. Prior research has found allogeneic transfusion to be associated with higher complication rates, ${ }^{2,3,33}$ including increases in the rates of surgical site infections, ${ }^{4}$ thromboembolic events, ${ }^{5}$ and kidney injuries. ${ }^{6}$ Additionally, the use of allogeneic blood products is associated with significant increases in cost, reported to average between $\$ 526$ per patient for anterior lumbar cases and $\$ 1980$ per

ABBREVIATIONS aPTT = activated partial thromboplastin time; ASA = American Society of Anesthesiologists; $A S D=$ adult spinal deformity; ASD-S = adult spinal deformity-surgical; EACA = $\varepsilon$-aminocaproic acid; INR = international normalized ratio; $\mathrm{MCV}=$ mean corpuscular volume; $\mathrm{PRBC}=$ packed red blood cell; RCT = randomized controlled trial; $T X A=$ tranexamic acid.

SUBMITTED December 8, 2019. ACCEPTED January 21, 2020.

INCLUDE WHEN CITING Published online March 20, 2020; DOI: 10.3171/2020.1.SPINE191464.

${ }^{*}$ J.E. and Z.P. contributed equally to this work. 
patient for posterior lumbar fusion operations. ${ }^{7}$ To this end, prior hospital-level cost analyses have found that procedural modifications based on the increased use of evidence-based transfusion practice may reduce costs associated with allogeneic transfusion by up to $\$ 2.1$ million per year for large academic centers. ${ }^{3,8}$

Because of the benefits of evidence-based treatment modifications on both outcomes and costs, there is clear incentive for health systems to identify means of reducing transfusion rates in spine surgery. Several interventions have been studied to achieve this goal, including the use of alternative surgical positioning designed to reduce intraabdominal pressure (e.g., Jackson frame, Wilson frame), intraoperative cell salvage, controlled hypotensive anesthesia, topical hemostatic agents, and systemic antifibrinolytic agents such as tranexamic acid (TXA). Of these, the best evidence exists for the use of antifibrinolytics, with multiple small randomized trials showing reductions in intraoperative and total blood loss with use of TXA. ${ }^{9-11}$

The question remains, however, regarding how the cost of TXA compares to the cost savings from decreased transfusions and complications. The objective of the present study was to examine this question in a cohort of patients undergoing elective lumbar surgery for degenerative spinal pathologies.

\section{Methods}

After obtaining Institutional Review Board approval (IRB00200167), we retrospectively reviewed all patients who underwent elective lumbar or thoracolumbar surgery at a tertiary care center between July 1, 2016, and November 23,2018 . We defined lumbar surgery as all procedures restricted to the levels between T11 and the pelvis. Inclusion criteria were the following: 1) age 18 years or older, 2) degenerative pathology as the indication, and 3) complete preoperative laboratory work available, including a complete blood count, coagulation testing, and a comprehensive metabolic panel, performed within 30 days of surgery. Patients were excluded if they had active signs of infection (defined as meeting systemic inflammatory response syndrome criteria) or if the primary indication for surgery was tumor, infection, trauma, or early-onset deformity (defined as coronal or sagittal deformity diagnosed before age 18 years).

Patients who received TXA made up the treatment group, and these patients were matched with non-TXA patients by age, sex, BMI, American Society of Anesthesiologists (ASA) physical status class, and surgical invasiveness score ${ }^{12}$ to make up the control group. For the surgical invasiveness score, we used the adult spinal deformitysurgical (ASD-S) score, as it has been shown to outperform the Surgical Invasiveness Index in terms of predicting blood loss. ${ }^{12}$ For included patients, we gathered data on the surgery performed, preoperative blood counts (hemoglobin, hematocrit, mean corpuscular volume [MCV], platelet count), preoperative coagulation parameters (activated partial thromboplastin time [aPTT], international normalized ratio [INR]), packed red blood cell (PRBC) units transfused, TXA quantity used, costs associated with
TXA use and PRBC transfusions, and postoperative data (length of hospital stay, occurrence of thrombotic events). The dose of TXA given varied; however, the typical dose was $10 \mathrm{mg} / \mathrm{kg}$ intravenously (IV) as a loading dose and $1-3 \mathrm{mg} / \mathrm{kg} / \mathrm{hr}$ IV as a continuous infusion throughout surgery. Our institution utilized a PRBC transfusion protocol with a hemoglobin threshold of $7 \mathrm{~g} / \mathrm{dL}$; however, a higher threshold was used for certain indications (signs of cerebral or cardiac ischemia, active bleeding/hemodynamically unstable, symptomatic anemia).

The primary outcome of the study was the combined cost of TXA and blood transfusions (total hemostasis cost). The secondary endpoints were cost per patient associated with TXA use, mean intraoperative blood loss per patient, numbers per patient of intraoperative and total PRBC units given, in-hospital thrombotic events, and length of hospital stay. To examine the influence of construct length on the cost-effectiveness of TXA use, we arbitrarily stratified patients into short-length (1-4 instrumented levels) and moderate-length (5-8 instrumented levels) constructs.

Costs associated with PRBC transfusion were obtained from our institutional blood bank, which is responsible for dispensing blood products. We utilized an activity-based design (or "bottom-up" design) to track costs, which involved totaling the specific costs of TXA and blood transfusion for each patient to compare across groups. This is in opposition to the "traditional costing" design, which solely analyzes total cost and assumes individual costs of different patients to be equal. Costs of PRBC transfusion are reported per unit, which includes the direct acquisition cost (\$200) as well as the indirect costs associated with managing the blood from donor to recipient (e.g., type and screen, crossmatching, cold storage, transport of unit from blood bank). These indirect costs are reported to bring up the total cost for 1 PRBC to 4-fold higher than the direct acquisition costs (i.e., \$800). ${ }^{10}$ Both direct acquisition costs and indirect costs were included in this study. TXA was supplied from the hospital pharmacy, and cost information was obtained from the critical care and surgery pharmacy. Costs of TXA use are reported per milligram delivered ( $\$ 12.11$ per $1000 \mathrm{mg}$ based on the wholesale acquisition cost as of October 1, 2019). Based upon the available records, it was not possible to apportion the costs associated with operating room time or anesthesia staff time to either intervention and these were excluded from the analysis.

\section{Statistical Analysis}

All data were gathered using Microsoft Excel 2018 and analyzed using JMP v14 (SAS Institute). Descriptive statistics are presented as mean \pm standard deviation for continuous variables and as proportions for categorical variables. Direct comparisons between patients receiving TXA and those not receiving TXA were performed using the Wilcoxon rank-sum test for continuous outcomes (e.g., blood loss, costs) and the McNemar-Bowker test for dichotomous outcomes (e.g., receipt of PRBC transfusion); chi-square tests were used for comparing categorical outcomes between groups. To analyze cost data for outliers, the Huber model was utilized, with a robust outlier detection function. 


\section{Results}

Total Cohort

Our search identified 1353 patients, of whom 68 received intraoperative TXA. A same-sized control group was then created by matching patients based on age, sex, BMI, ASA physical status class, and surgical invasiveness score. The preoperative, intraoperative, and postoperative variables were then compared between the TXA and non-TXA groups (Table 1). There were no significant differences between groups in terms of demographic factors, preoperative laboratory values, and surgical duration. Similarly, the average surgical invasiveness scores of each group did not significantly differ, and both groups had a mean score of approximately $19(19.4 \pm 8.1$ vs $18.9 \pm 7.3, p=0.7)$. Significantly more patients in the TXA group underwent pelvic fixation than in the non-TXA group $(57.4 \%$ vs $25.0 \%$, p < 0.001 ), likely due to surgeon expectations of greater blood loss with pelvic fixation. There was no difference in interbody use between groups ( $39.7 \%$ vs $44.1 \%, p=0.6)$. Regarding surgical approach, all patients in the TXA group underwent a posterior approach, while 4 patients $(5.9 \%)$ in the non-TXA group underwent an anterior approach, with the remaining being posterior $(\mathrm{p}=0.017)$. The mean number of PRBC units transfused intraoperatively was not significantly different between the TXA and non-TXA groups $(1.2 \pm 2.0$ units vs $1.5 \pm 2.0$ units, $p=0.341)$. The group of patients who received TXA had significantly lower intraoperative blood loss $(1039 \pm 737$ vs $1437 \pm 1032 \mathrm{~mL}, \mathrm{p}=$ $0.01)$ and received a lower volume of crystalloid (2821 \pm 1023 vs $3342 \pm 1204 \mathrm{~mL}, \mathrm{p}=0.007)$. The group receiving TXA had nonsignificant differences regarding drop in hemoglobin $(-23.8 \%$ vs $-28.0 \%, \mathrm{p}=0.190)$ and hematocrit $(-24.5 \%$ vs $-28.5 \%, \mathrm{p}=0.182)$ based on preoperative and postoperative day 1 laboratory values. We found no difference in estimated blood loss when comparing posterior to anterior approaches, respectively (1256 \pm 912 vs $1087 \pm$ $1130 \mathrm{~mL}, \mathrm{p}=0.511$ ). There were no significant differences in terms of postoperative thromboembolic events, hospitalization duration, or probability of being discharged home versus to rehabilitation. No significant difference was seen in total PRBC units transfused over the entire hospital stay.

At a wholesale acquisition cost of $\$ 12.11$ per 1000 $\mathrm{mg} / 10 \mathrm{~mL}$ vial (as of October 1, 2019), TXA use amounted to $\$ 25.81$ per patient (Table 2). Of patients who did not receive TXA, 54.4\% required at least 1 blood transfusion throughout their hospital stay, compared to $47.1 \%$ of the TXA patients. Taking into consideration all PRBC units given, the non-TXA group received an average of $2.3 \pm$ 2.4 units in their hospital stay, compared to $1.6 \pm 2.0$ units in the TXA group $(\mathrm{p}=0.1)$. In considering the "acquisition-only cost," which was $\$ 200.00$ at our institution, this equated to an average PRBC cost of $\$ 452.94$ for the nonTXA group and $\$ 323.52$ for the TXA group. However, when TXA cost was added to the acquisition-only PRBC cost in the TXA group, the net costs between groups were not significantly different $(\mathrm{p}=0.5$; Table 2$)$. No cost outliers were identified in either cohort.

\section{Short-Length Construct Cohort}

After stratification by construct length, the short-length
TABLE 1. Data for 136 patients undergoing lumbar spine surgery with or without treatment with intraoperative TXA

\begin{tabular}{|c|c|c|c|}
\hline & No TXA $(n=68)$ & $\operatorname{TXA}(n=68)$ & $p$ Value \\
\hline \multicolumn{4}{|l|}{ Demographic factors } \\
\hline Age, yrs & $63 \pm 12$ & $63 \pm 14$ & 0.817 \\
\hline Male sex & $36(52.9 \%)$ & $36(52.9 \%)$ & 1.000 \\
\hline $\mathrm{BMI}, \mathrm{kg} / \mathrm{m}^{2}$ & $30.5 \pm 6.1$ & $30.3 \pm 6.4$ & 0.797 \\
\hline $\mathrm{CCl}$ score & $1.2 \pm 1.5$ & $1.2 \pm 1.6$ & 0.956 \\
\hline Hypertension & $43(63.2 \%)$ & $36(52.9 \%)$ & 0.228 \\
\hline Coagulopathy & $2(2.9 \%)$ & $5(7.4 \%)$ & 0.237 \\
\hline $\begin{array}{l}\text { ASA physical status } \\
\text { class }\end{array}$ & $2.6 \pm 0.6$ & $2.5 \pm 0.6$ & 0.654 \\
\hline \multicolumn{4}{|l|}{ Preop lab values } \\
\hline Hemoglobin, mg/dL & $13.6 \pm 1.5$ & $13.1 \pm 1.9$ & 0.099 \\
\hline Hematocrit, \% & $41.3 \pm 4.0$ & $40.3 \pm 4.3$ & 0.195 \\
\hline Platelet count, $\times 10^{3} / \mu \mathrm{L}$ & $246.2 \pm 75.7$ & $254.4 \pm 82.3$ & 0.557 \\
\hline Mean platelet vol, fL & $10.2 \pm 1.1$ & $10.1 \pm 2.2$ & 0.777 \\
\hline aPTT, secs & $25.7 \pm 4.8$ & $26.0 \pm 3.7$ & 0.671 \\
\hline INR & $1.02 \pm 0.18$ & $1.02 \pm 0.06$ & 0.811 \\
\hline \multicolumn{4}{|l|}{ Intraop factors } \\
\hline ASD-S score & $19.4 \pm 8.1$ & $18.9 \pm 7.3$ & 0.673 \\
\hline $\begin{array}{l}\text { Construct length, no. } \\
\text { of levels }\end{array}$ & $4 \pm 1$ & $5 \pm 2$ & 0.051 \\
\hline Pelvic fixation & $17(25.0 \%)$ & $39(57.4 \%)$ & $<0.001$ \\
\hline Interbody & $30(44.1 \%)$ & $27(39.7 \%)$ & 0.602 \\
\hline Surgical approach & & & 0.017 \\
\hline Posterior & $64(94.1 \%)$ & $68(100.0 \%)$ & \\
\hline Anterior & $4(5.9 \%)$ & $0(0.0 \%)$ & \\
\hline Blood loss, mL & $1437 \pm 1032$ & $1039 \pm 737$ & 0.010 \\
\hline MAP, $\mathrm{mm} \mathrm{Hg}$ & $82 \pm 7$ & $82 \pm 1$ & 0.591 \\
\hline Crystalloid, mL & $3342 \pm 1204$ & $2821 \pm 1023$ & 0.007 \\
\hline Colloid, mL & $556 \pm 504$ & $625 \pm 473$ & 0.409 \\
\hline Op duration, mins & $316 \pm 101$ & $322 \pm 95$ & 0.715 \\
\hline PRBC units given & $2 \pm 2$ & $1 \pm 2$ & 0.341 \\
\hline \multicolumn{4}{|l|}{ Postop day 1 lab values } \\
\hline Hemoglobin, mg/dL & $9.7 \pm 1.3$ & $10.1 \pm 1.4$ & 0.169 \\
\hline$\Delta$ Hemoglobin, $\%$ & $-28.0 \pm 10.4$ & $-23.8 \pm 12.5$ & 0.190 \\
\hline Hematocrit, \% & $29.3 \pm 4.0$ & $30.3 \pm 4.2$ & 0.165 \\
\hline$\Delta$ Hematocrit, $\%$ & $-28.5 \pm 10.6$ & $-24.5 \pm 11.3$ & 0.182 \\
\hline \multicolumn{4}{|l|}{ Whole hospitalization } \\
\hline Total PRBC units given & $2.15 \pm 2.44$ & $1.72 \pm 2.12$ & 0.106 \\
\hline DVT/PE/DIC & $3(4.4)$ & $0(0)$ & 0.154 \\
\hline Length of stay, days & $6.17 \pm 4.79$ & $5.89 \pm 2.93$ & 0.683 \\
\hline Discharge to home & $53(77.9)$ & $52(76.5)$ & 0.788 \\
\hline
\end{tabular}

$\mathrm{CCl}=$ Charlson Comorbidity Index; $\mathrm{DIC}=$ disseminated intravascular coagulation; DVT = deep venous thrombosis; $\mathrm{MAP}=$ mean arterial pressure; $\mathrm{PE}=$ pulmonary embolism.

Values are presented as number (\%) of patients unless otherwise indicated. Mean values are presented \pm SD. Significance level $a=0.05$. Boldface type indicates statistical significance. 
TABLE 2. Pricing of TXA and transfused PRBC units in patients with or without intraoperative TXA

\begin{tabular}{|c|c|c|c|}
\hline & No TXA $(n=68)$ & TXA $(n=68)$ & p Value \\
\hline Transfusion-treated pts & $31(45.6 \%)$ & $37(54.4 \%)$ & 0.391 \\
\hline PRBC units given during hospital stay, mean & 2.3 & 1.6 & 0.106 \\
\hline TXA cost per pt in cohort, mean* & $\$ 0$ & $\$ 25.81$ & - \\
\hline PRBC unit acquisition-only cost throughout hospital stay, mean per pt $\dagger$ & $\$ 452.94$ & $\$ 323.52$ & 0.106 \\
\hline PRBC unit net acquisition-only cost + TXA cost throughout hospital stay, mean per pt $†$ & $\$ 452.94$ & $\$ 349.34$ & 0.532 \\
\hline PRBC unit total cost throughout hospital stay, mean per pt $\ddagger$ & $\$ 1811.76$ & $\$ 1294.12$ & 0.106 \\
\hline PRBC unit net total cost + TXA cost throughout hospital stay, mean per pt & $\$ 1811.76$ & $\$ 1319.93$ & 0.532 \\
\hline $\begin{array}{l}\text { pt = patient. } \\
\text { Significance level } \alpha=0.05 . \\
{ }^{*} \text { Cost of } 1 \text { TXA vial }(1000 \mathrm{mg} / 10 \mathrm{~mL}) \$ 12.106 . \\
\dagger \text { Blood acquisition cost of } 1 \text { unit of PRBCs } \$ 200.00 \text {. }\end{array}$ & & & \\
\hline
\end{tabular}

cohort was made up of 40 patients in the non-TXA group and 34 patients in the TXA group (Table 3). There were no significant differences between demographic factors, preoperative laboratory values, or surgical durations between the groups. Similarly, the surgical invasiveness score of each group did not significantly differ between the nonTXA and TXA groups (16.1 vs 14.5, p = 0.3). Significantly more patients in the TXA group underwent pelvic fixation compared to the non-TXA group (20.6\% vs 5.0\%, p = $0.034)$. There was no difference in interbody use between groups $(50.0 \%$ vs $62.5 \%, \mathrm{p}=0.3)$. Regarding surgical approach, all patients in the TXA group underwent a posterior approach, while 4 patients $(10.0 \%)$ in the non-TXA group underwent the anterior approach with the remaining being posterior $(\mathrm{p}=0.025)$. Mean PRBC units transfused intraoperatively did not significantly differ between the TXA and non-TXA groups $(0.7 \pm 1.4$ vs $0.6 \pm 1.4$ units, $\mathrm{p}=0.952$ ). The group that received TXA had significantly lower intraoperative blood loss $(689 \pm 528$ vs $1021 \pm 661$ $\mathrm{mL}, \mathrm{p}=0.008$ ). Furthermore, the group receiving TXA had a significantly smaller drop in hemoglobin $(-19.8 \%$ vs $-28.7 \%, \mathrm{p}=0.006)$ and hematocrit $(-20.6 \%$ vs $-28.7 \%$, $\mathrm{p}=0.014)$ based on preoperative and postoperative day 1 laboratory values. We found no difference in estimated blood loss when comparing posterior to anterior approaches, respectively $(858 \pm 593$ vs $1087 \pm 1130 \mathrm{~mL}, \mathrm{p}=$ 0.925). There was no significant difference in total PRBC units given throughout the hospital stay between the TXA and non-TXA groups ( 0.8 vs 1.1 days, $\mathrm{p}=0.2$ ). There were also no significant differences in terms of postoperative thromboembolic events, hospitalization duration, or probability of being discharged home versus to rehabilitation.

Patients in the short-length construct group receiving TXA had an average TXA cost of \$24.21 (Table 4). Of patients who did not receive TXA, 36.6\% required at least 1 blood transfusion throughout their hospital stay, compared to $26.5 \%$ of the TXA patients $(\mathrm{p}=0.348)$. In considering the "acquisition-only cost," this equated to an average PRBC cost of \$214.29 for the non-TXA group and \$164.71 for the TXA group. However, when TXA cost was added to the acquisition-only PRBC cost in the TXA group, the net costs between groups were not significantly different $(\mathrm{p}=0.3)($ Table 4$)$.

\section{Long-Length Construct Cohort}

After stratification by construct length, the long-length cohort was made up of 28 patients in the non-TXA group and 34 patients in the TXA group (Table 5). There were no significant differences between demographic factors, preoperative laboratory values, or surgical duration between the groups. Similarly, the surgical invasiveness score of each group did not significantly differ between the nonTXA and TXA groups $(25.1 \pm 7.5$ vs $23.5 \pm 5.6, \mathrm{p}=0.5)$. Significantly more patients in the TXA group underwent pelvic fixation compared to the non-TXA group $(94.1 \%$ vs $53.6 \%, \mathrm{p}<0.001)$. There was no difference in interbody use between groups (29.4\% vs $17.9 \%, \mathrm{p}=0.3)$. All patients with long-length constructs underwent posterior approaches. The group that received TXA had significantly lower intraoperative blood loss $(1402 \pm 756$ vs $2118 \pm 1135 \mathrm{~mL}$, $\mathrm{p}$ $=0.007)$. The TXA group also received significantly fewer PRBC units intraoperatively than the non-TXA group (1.5 \pm 2.0 vs $3.1 \pm 2.0$ units, $p=0.001$ ). There were no significant differences between drops in hemoglobin or hematocrit between preoperative and postoperative day 1 values. Fewer PRBC units were given to the TXA group over the total hospital stay $(2.4 \pm 2.1$ vs $4.0 \pm 2.5$ units, $\mathrm{p}=0.007)$. There were no significant differences in terms of postoperative thromboembolic events, hospitalization duration, or probability of being discharged home versus to rehabilitation.

Patients in the long-length construct group receiving TXA had an average TXA cost of $\$ 27.42$ (Table 4). Of patients who did not receive TXA, $81.5 \%$ required at least 1 blood transfusion, compared to $67.6 \%$ of the TXA patients $(p=0.27)$. Taking into consideration all PRBC units given, the non-TXA group received an average of 4.0 units in their hospital stay, compared to 2.4 units in the TXA group ( $\mathrm{p}=0.007)$. In considering the acquisition-only cost, this equated to an average PRBC cost of $\$ 838.46$ for the non-TXA group and $\$ 482.35$ for the TXA group ( $\mathrm{p}=$ 0.003 ). When TXA cost was added to the acquisition-only PRBC cost in the TXA group, the net cost savings with TXA was $\$ 328.69$ per patient $(\mathrm{p}=0.027)($ Table 4$)$.

\section{Discussion}

Transfusion of allogeneic blood products is associated 
TABLE 3. Data from subanalyses of 74 patients undergoing short-length construct (1-4 levels) lumbar spine surgery who were or were not treated with intraoperative TXA

\begin{tabular}{|c|c|c|c|}
\hline & No TXA $(n=40)$ & TXA $(n=34)$ & $p$ Value \\
\hline \multicolumn{4}{|l|}{ Demographic factors } \\
\hline Age, yrs & $60 \pm 13$ & $60 \pm 14$ & 0.753 \\
\hline Male sex & $20(48.8 \%)$ & $19(55.9 \%)$ & 0.540 \\
\hline BMI, $\mathrm{kg} / \mathrm{m}^{2}$ & $29.1 \pm 5.6$ & $30.0 \pm 5.4$ & 0.807 \\
\hline $\mathrm{CCl}$ score & $1.0 \pm 1.3$ & $1.3 \pm 1.7$ & 0.574 \\
\hline Hypertension & $22(53.7 \%)$ & $16(47.1 \%)$ & 0.569 \\
\hline Coagulopathy & $0(0 \%)$ & $3(8.8 \%)$ & 0.052 \\
\hline ASA physical status class & $2.4 \pm 0.5$ & $2.5 \pm 0.6$ & 0.665 \\
\hline \multicolumn{4}{|l|}{ Preop lab values } \\
\hline Hemoglobin, mg/dL & $13.8 \pm 1.5$ & $12.9 \pm 2.2$ & 0.156 \\
\hline Hematocrit, \% & $41.8 \pm 4.2$ & $40.4 \pm 4.5$ & 0.249 \\
\hline Platelet count, $\times 10^{3} / \mu \mathrm{L}$ & $243 \pm 75$ & $250 \pm 65$ & 0.411 \\
\hline Mean platelet vol, fL & $10.2 \pm 0.9$ & $9.4 \pm 1.7$ & 0.014 \\
\hline aPTT, secs & $25.8 \pm 5.8$ & $27.2 \pm 3.9$ & 0.761 \\
\hline INR & $0.99 \pm 0.17$ & $1.0 \pm 0.07$ & 0.784 \\
\hline \multicolumn{4}{|l|}{ Intraop factors } \\
\hline ASD-S score & $16.1 \pm 6.4$ & $14.5 \pm 5.8$ & 0.314 \\
\hline Construct length, no. of levels & $3 \pm 1$ & $3 \pm 1$ & 0.517 \\
\hline Pelvic fixation & $2(5.0 \%)$ & $7(20.6 \%)$ & 0.034 \\
\hline Interbody & $25(62.5 \%)$ & $17(50.0 \%)$ & 0.340 \\
\hline Surgical approach & & & 0.025 \\
\hline Posterior & $36(90.0 \%)$ & $68(100.0 \%)$ & \\
\hline Anterior & $4(10.0 \%)$ & $0(0.0 \%)$ & \\
\hline Blood loss, mL & $1021 \pm 661$ & $689 \pm 528$ & 0.008 \\
\hline MAP, $\mathrm{mm} \mathrm{Hg}$ & $81.7 \pm 7.2$ & $81.5 \pm 8.7$ & 0.833 \\
\hline Crystalloid, mL & $2968 \pm 958$ & $2448 \pm 782$ & 0.017 \\
\hline Colloid, $\mathrm{mL}$ & $531 \pm 506$ & $436 \pm 372$ & 0.607 \\
\hline Surgical duration, mins & $290.2 \pm 90.2$ & $271.0 \pm 73.2$ & 0.351 \\
\hline PRBC units given & $0.6 \pm 1.0$ & $0.7 \pm 1.4$ & 0.952 \\
\hline \multicolumn{4}{|l|}{ Post day 1 lab values } \\
\hline Hemoglobin, mg/dL & $9.8 \pm 1.4$ & $10.6 \pm 1.5$ & 0.040 \\
\hline$\Delta$ Hemoglobin, $\%$ & $-28.7 \pm 10.0$ & $-19.8 \pm 12.4$ & 0.006 \\
\hline Hematocrit, \% & $29.6 \pm 4.3$ & $32.0 \pm 4.3$ & 0.042 \\
\hline$\Delta$ Hematocrit, $\%$ & $-28.7 \pm 10.7$ & $-20.6 \pm 11.6$ & 0.014 \\
\hline \multicolumn{4}{|l|}{ Whole hospitalization } \\
\hline Total PRBCs given, units & $1.1 \pm 1.5$ & $0.8 \pm 1.6$ & 0.201 \\
\hline DVT/PE & $1(2.4 \%)$ & $0(0 \%)$ & 0.343 \\
\hline Length of stay, days & $5.0 \pm 2.3$ & $4.9 \pm 2.8$ & 0.762 \\
\hline Discharge to home & $35(85.4 \%)$ & $30(88.2 \%)$ & 0.746 \\
\hline
\end{tabular}

Values are presented as number (\%) of patients unless otherwise indicated. Mean values are presented \pm SD. Significance level $\alpha=0.05$. Boldface type indicates statistical significance.

with higher rates of patient complications ${ }^{2,8}$ and higher costs of care. ${ }^{8}$ There have been many interventions tested in the literature for their ability to reduce transfusion requirements, with varying degrees of reported success. Here we examined the impact of one intervention-TXA administration - to reduce intraoperative blood loss and decrease the costs associated with transfusion. We found in a subanalysis of this matched case-control study that patients who underwent long-length spine surgery and received intraoperative TXA had decreased intraoperative blood loss and a significant net cost benefit of \$328.69.

\section{Evidence for the Use of TXA in Spine Surgery}

Several studies have been performed examining the ef- 
TABLE 4. Data from subanalyses of TXA pricing and units of transfused PRBCs for short- and long-length constructs with or without intraoperative TXA

\begin{tabular}{|c|c|c|c|c|c|c|}
\hline & \multicolumn{3}{|c|}{ Short-Length Construct (1-4 levels) } & \multicolumn{3}{|c|}{ Long-Length Construct (5-8 levels) } \\
\hline & $\begin{array}{l}\text { No TXA } \\
(n=40)\end{array}$ & $\begin{array}{c}\text { TXA } \\
(n=34)\end{array}$ & $\begin{array}{c}p \\
\text { Value }\end{array}$ & $\begin{array}{l}\text { No TXA } \\
(n=28)\end{array}$ & $\begin{array}{c}\text { TXA } \\
(n=34)\end{array}$ & $\begin{array}{c}p \\
\text { Value }\end{array}$ \\
\hline Transfusion-treated pts & $15(36.6 \%)$ & $9(26.5 \%)$ & 0.348 & $22(81.5 \%)$ & $23(67.7 \%)$ & 0.27 \\
\hline PRBC units during hospital stay, mean & 1.1 & 0.8 & 0.201 & 4.0 & 2.4 & 0.007 \\
\hline TXA cost per pt in cohort, mean* & $\$ 0$ & $\$ 24.21$ & - & $\$ 0$ & $\$ 27.42$ & - \\
\hline PRBC unit acquisition-only cost throughout hospital stay, mean per pt $†$ & $\$ 214.29$ & $\$ 164.71$ & 0.230 & $\$ 838.46$ & $\$ 482.35$ & 0.003 \\
\hline $\begin{array}{l}\text { PRBC unit net acquisition-only cost + TXA cost throughout hospital stay, } \\
\text { mean per pt } \dagger\end{array}$ & $\$ 214.29$ & $\$ 188.92$ & 0.322 & $\$ 838.46$ & $\$ 509.77$ & 0.027 \\
\hline PRBC unit total cost throughout hospital stay, mean per pt & $\$ 857.14$ & $\$ 658.82$ & 0.230 & $\$ 3353.85$ & $\$ 1929.41$ & 0.003 \\
\hline PRBC unit net total cost + TXA cost throughout hospital stay, mean per pt & $\$ 857.14$ & $\$ 683.03$ & 0.322 & $\$ 3353.85$ & $\$ 1956.83$ & 0.027 \\
\hline
\end{tabular}

Significance level $\alpha=0.05$. Boldface type indicates statistical significance.

${ }^{*}$ Cost of 1 TXA vial $(1000 \mathrm{mg} / 10 \mathrm{~mL}) \$ 12.106$.

$\dagger$ Blood acquisition cost of 1 unit of PRBCs $\$ 200.00$.

$\ddagger$ Total cost (including acquisition cost + overhead costs) of 1 unit of PRBCs, including materials, labor, third-party services, approximately $\$ 800.00 .{ }^{10}$

ficacy of TXA in reducing blood loss in patients undergoing spine surgery. A double-blinded randomized controlled trial (RCT) conducted by Sethna and colleagues, ${ }^{13}$ evaluated intraoperative blood loss in 44 children undergoing scoliosis surgery who received either TXA $(100 \mathrm{mg} /$ $\mathrm{kg}$ bolus followed by $10 \mathrm{mg} / \mathrm{kg} / \mathrm{hr}$ infusion) or normal saline. The authors observed a $41 \%$ lower blood loss among TXA-treated patients along with a $34 \%$ reduction in transfusions, though this difference was not statistically significant due to large variability in the amount transfused. No differences were found in complication rates or rates of transfusion of other blood products. Similar findings were subsequently reported by Wong et al.. ${ }^{14}$ who conducted a multicenter double-blinded RCT of 147 adults undergoing thoracolumbar fusion randomized to either TXA (10 $\mathrm{mg} / \mathrm{kg}$ bolus followed by $1 \mathrm{mg} / \mathrm{kg} / \mathrm{hr}$ infusion) or normal saline. Patients receiving TXA experienced 25\% lower intraoperative blood loss, though this did not translate to a significant difference in the rate of PRBC transfusion. There were no differences in complication rates between the groups, suggesting TXA use could reduce surgical bleeding without a concordant increase in risk.

Wang et al. ${ }^{11}$ examined the effect of TXA use in an unblinded RCT of 60 patients undergoing lumbar fusion for degenerative pathologies who received either TXA $(15 \mathrm{mg} /$ $\mathrm{kg}$ bolus) or normal saline. In contrast to prior studies, the authors found no significant difference in intraoperative blood loss between the treatment groups; however, TXA use led to a $13 \%$ decrease in postoperative bleeding and only a bolus was given, with no continuous infusion. The same year, Elwatidy et al. ${ }^{15}$ reported the results of a doubleblinded RCT of 64 patients undergoing spine surgeries with "expected significant blood loss." Use of TXA ( 2 g loading dose followed by $100 \mathrm{mg} / \mathrm{hr}$ continuous infusion) was associated with a $49 \%$ reduction in intraoperative blood loss and an $80 \%$ reduction in the rate of allogeneic blood transfusion without an increase in perioperative complications.

Peters and colleagues ${ }^{16}$ conducted a double-blinded RCT of 51 patients undergoing posterior fusion of $\geq 5$ spinal levels for adult spinal deformity (ASD) who were ran- domized to receive $\varepsilon$-aminocaproic acid (EACA), TXA, or saline (control). They found that EACA use was associated with a significant reduction in blood loss relative to control (approximately $100 \mathrm{~mL} / \mathrm{level}$ ); TXA use trended toward lower blood loss, but the difference was not significant. EACA was also found to decrease postoperative transfusion risk relative to TXA. In a later 3-armed double-blinded RCT comparing EACA, TXA, and placebo in 125 adolescents undergoing surgery for adolescent idiopathic scoliosis, Verma and colleagues ${ }^{29}$ found that both TXA and EACA reduced intraoperative blood loss relative to placebo. Similar to the earlier study, EACA produced a greater reduction in intraoperative blood loss relative to control; however, only TXA was found to significantly reduce postoperative drainage and total blood loss relative to control.

Though the majority of the studies of TXA have focused on its ability to decrease blood loss in large deformity operations, Kushioka et al. ${ }^{17}$ recently provided evidence that it may provide significant benefits even in smaller cases. In their case-control study of 60 patients who underwent single-level posterior lumbar interbody fusion, they found that TXA use was associated with a $39 \%$ reduction in intraoperative blood loss, $52 \%$ reduction in postoperative blood loss, and $47 \%$ decrease in total blood loss. Neither group required allogeneic blood transfusion, and complication rates were similar in both.

Meta-analyses of the available literature in spinal surgery have echoed the results of these trials. Reviews by McNicol et al., ${ }^{18}$ Cheriyan et al., ${ }^{19}$ Wang et al., ${ }^{20}$ Yuan et al. ${ }^{21} \mathrm{Li}$ et al. ${ }^{22}$ and Zhong et al. ${ }^{23}$ have found antifibrinolytic use to decrease intraoperative blood loss by more than $20 \%$ and decrease transfusion requirements by up to $45 \% .^{21}$ TXA specifically is associated with a $33 \%-45 \%$ reduction in the odds of requiring a transfusion and a $28 \%$ decrease in intraoperative blood loss without noted increases in perioperative complication risk. ${ }^{19,21}$ Additionally, these reviews have found antifibrinolytic use to significantly reduce postoperative blood loss, which is an often neglected contribution making up to $30 \%$ of total blood loss. ${ }^{24}$ 
TABLE 5. Data from subanalyses for 62 patients undergoing long-length construct (5-8 levels) lumbar spine surgery treated with or without intraoperative TXA

\begin{tabular}{|c|c|c|c|}
\hline & No TXA $(n=28)$ & $\operatorname{TXA}(n=34)$ & $p$ Value \\
\hline \multicolumn{4}{|l|}{ Demographic factors } \\
\hline Age, yrs & $65.9 \pm 9.8$ & $66.7 \pm 13.1$ & 0.467 \\
\hline Male sex & $16(59.3 \%)$ & $16(47.1 \%)$ & 0.342 \\
\hline $\mathrm{BMI}, \mathrm{kg} / \mathrm{m}^{2}$ & $33.1 \pm 6.2$ & $30.7 \pm 7.4$ & 0.104 \\
\hline $\mathrm{CCl}$ score & $1.6 \pm 1.7$ & $1.1 \pm 1.4$ & 0.130 \\
\hline Hypertension & $20(74.1 \%)$ & $19(55.9 \%)$ & 0.138 \\
\hline Coagulopathy & $2(7.4 \%)$ & $2(5.9 \%)$ & 0.812 \\
\hline ASA physical status class & $2.8 \pm 0.5$ & $2.6 \pm 0.6$ & 0.134 \\
\hline \multicolumn{4}{|l|}{ Preop lab values } \\
\hline Hemoglobin, mg/dL & $13.3 \pm 1.4$ & $13.4 \pm 1.5$ & 0.582 \\
\hline Hematocrit, \% & $40.4 \pm 3.5$ & $40.6 \pm 3.7$ & 0.725 \\
\hline Platelet count, $\times 10^{3} / \mu \mathrm{L}$ & $248 \pm 81$ & $260 \pm 98$ & 0.480 \\
\hline Mean platelet vol, fL & $10.3 \pm 1.5$ & $10.8 \pm 2.3$ & 0.915 \\
\hline aPTT, secs & $25.6 \pm 3.0$ & $25.0 \pm 3.3$ & 0.372 \\
\hline INR & $1.06 \pm 0.20$ & $1.03 \pm 0.07$ & 0.864 \\
\hline \multicolumn{4}{|l|}{ Intraop factors } \\
\hline ASD-S score & $25.1 \pm 7.5$ & $23.5 \pm 5.6$ & 0.459 \\
\hline Construct length, no. of levels & $6 \pm 1$ & $6 \pm 1$ & 0.070 \\
\hline Pelvic fixation & $15(53.6 \%)$ & $32(94.1 \%)$ & $<0.001$ \\
\hline Interbody & $5(17.9 \%)$ & $10(29.4 \%)$ & 0.322 \\
\hline Posterior approach & $28(100.0 \%)$ & $34(100.0 \%)$ & 1.0 \\
\hline Blood loss, $\mathrm{mL}$ & $2118 \pm 1135$ & $1402 \pm 756$ & 0.007 \\
\hline MAP, $\mathrm{mm} \mathrm{Hg}$ & $81.2 \pm 5.9$ & $81.9 \pm 9.5$ & 0.841 \\
\hline Crystalloid, mL & $3958 \pm 1358$ & $3211 \pm 1110$ & 0.020 \\
\hline Colloid, $\mathrm{mL}$ & $601 \pm 515$ & $830 \pm 479$ & 0.087 \\
\hline Op duration, mins & $373.1 \pm 94.7$ & $375.3 \pm 86.6$ & 0.979 \\
\hline Intraop PRBC units & $3.1 \pm 2.0$ & $1.5 \pm 2.0$ & 0.001 \\
\hline \multicolumn{4}{|l|}{ Postop day 1 lab values } \\
\hline Hemoglobin, mg/dL & $9.6 \pm 1.0$ & $9.6 \pm 1.1$ & 0.723 \\
\hline$\Delta$ Hemoglobin, $\%$ & $-26.9 \pm 10.8$ & $-27.9 \pm 11.3$ & 0.473 \\
\hline Hematocrit, \% & $28.7 \pm 3.3$ & $28.7 \pm 3.5$ & 0.623 \\
\hline$\Delta$ Hematocrit, $\%$ & $-28.2 \pm 10.4$ & $-29.0 \pm 10.4$ & 0.614 \\
\hline \multicolumn{4}{|l|}{ Whole hospitalization } \\
\hline Total PRBC units & $4.0 \pm 2.5$ & $2.4 \pm 2.1$ & 0.007 \\
\hline DVT/PE & $2(7.4 \%)$ & $0(0 \%)$ & 0.574 \\
\hline Length of stay, days & $8.9 \pm 7.2$ & $6.9 \pm 2.8$ & 0.971 \\
\hline Discharge to home & $17(62.9 \%)$ & $21(61.8 \%)$ & 0.857 \\
\hline
\end{tabular}

\section{Prior Examinations of Cost Savings Associated With Antifibrinolytic Use}

The main objective of the present study was to determine whether TXA is a cost-effective intervention among patients being treated for degenerative spine pathologies. Evidence from the orthopedic literature suggests that TXA can result in cost savings of at least $\$ 130$ per patient for those undergoing total hip arthroplasty, with savings more than 4-fold higher in revision surgical cases..$^{25,26}$ Furthermore, an analysis by the United Kingdom National
Clinical Guideline Centre found TXA to be equivalent or superior to all other interventions for reducing perioperative blood loss. ${ }^{27}$ Use of oral versus intravenous formulations is suggested to further increase cost savings without significantly changing intraoperative blood loss. ${ }^{28}$

Given that intraoperative blood losses are often similar between different types of lumbar spine surgeries, it is likely that cost savings would be similar to those demonstrated here. For larger spine procedures, the potential cost savings are most likely higher, and although such an 
investigation was beyond the scope of the present study, one is able to extrapolate and assume that cost savings may increase as the invasiveness of the surgery increases (i.e., deformity and tumor cases). ${ }^{1}$

\section{Merits of TXA Compared to Other Fibrinolytics}

Though Peters et al. ${ }^{16}$ found that EACA reduces blood loss relative to TXA, other studies from the spine ${ }^{18,20,22,29}$ and arthroplasty literature ${ }^{30-32}$ have either found no difference between the two antifibrinolytics or superior outcomes in those treated with TXA. Some authors have described EACA as less costly,,$^{31,32}$ but this likely depends upon the formulary and purchasing power of each surgeon's institution and is beyond the scope of the present discussion.

One concern with the use of antifibrinolytics is the creation of a prothrombotic state in the postoperative period. While physiologically there is some merit to this concern, it has not been supported in the literature. A $2011 \mathrm{Co}-$ chrane meta-analysis ${ }^{9}$ including more than 25,000 patients in both cardiac and noncardiac surgical fields found that TXA use was associated with a nearly $40 \%$ reduction in PRBC transfusion risk across all patients and a 51\% reduction for those undergoing orthopedic surgery. In agreement with the present study, these authors found no difference between TXA-treated patients and controls in terms of clinically evident venous thromboembolic event or prolonged hospitalization. ${ }^{9}$ However, it is possible that subclinical thrombotic events were missed because prospective surveillance was not included. For this reason, careful patient histories can attune physicians to whether patients are at high risk for thromboembolism, and this can inform the decision to use or not use TXA.

\section{Study Limitations}

The present study is not without limitations. Noted limitations include that it is a single-institution, retrospective study. As such, we are unable to infer a causal relationship between TXA use and cost savings, though the concordance of our findings with those in the arthroplasty and scoliosis literature suggests that such a relationship may exist. Additionally, our results are subject to institutionspecific factors, which could potentially limit their generalizability. This includes potential selection bias when choosing which patients to give TXA, which is evidenced by our finding that patients undergoing pelvic fixation (high expected blood loss) were more often treated with TXA. Furthermore, our substratification by construct length led to slightly different sample sizes between groups; however, Tables 3 and 5 show that there were no significant differences regarding demographic or operative data, so these groups remained cohort matched rather than individually matched. It is also important to note that our stratification into construct length was arbitrary in order to allow for similar sample sizes, and having greater or lesser than 4 instrumented levels does not objectively correspond to a "long" or "short" construct, respectively. The present cohort also excluded patients with active infection or tumor, and as these patients are often at high risk for thrombotic events, the present findings cannot be generalized outside of patients with degenerative spine indications. While similar evidence exists supporting the use of TXA in longsegment fusions for adolescent idiopathic scoliosis, there remains little to no evidence regarding the merits of TXA in adult thoracolumbar deformity and cervical spine operations. Further research is necessary to determine whether similar benefits are observed in this population. One additional limitation is the ideal dosing of TXA, which to date is poorly understood. Given that TXA administration to reduce bleeding and transfusion is considered "off-label" use, rigorous studies looking at dosing regimens have yet to be performed. Our cohort comprised those patients receiving relatively low-dose TXA, so higher doses may yield different results.

\section{Conclusions}

We found that in long-segment spinal fusions, TXA use is associated with decreased intraoperative blood loss and a significant cost benefit. Furthermore, we found that use of TXA in short-construct surgery led to no additional net costs. With the increasing emphasis put on value-based care interventions, use of TXA may represent one mechanism for decreasing total care costs, particularly in the cases of larger spine constructs.

\section{References}

1. iData Research. Spinal Implants Market Analysis. iData Research; 2019. https://idataresearch.com/product/spinalimplants-market/. Accessed February 6, 2020.

2. Aoude A, Nooh A, Fortin M, et al. Incidence, predictors, and postoperative complications of blood transfusion in thoracic and lumbar fusion surgery: an analysis of 13,695 patients from the American College of Surgeons National Surgical Quality Improvement Program database. Global Spine J. 2016;6(8):756-764.

3. Purvis TE, Goodwin CR, De la Garza-Ramos R, et al. Effect of liberal blood transfusion on clinical outcomes and cost in spine surgery patients. Spine J. 2017;17(9):1255-1263.

4. Rohde JM, Dimcheff DE, Blumberg N, et al. Health careassociated infection after red blood cell transfusion: a systematic review and meta-analysis. JAMA. 2014;311(13):13171326.

5. Goel R, Patel EU, Cushing MM, et al. Association of perioperative red blood cell transfusions with venous thromboembolism in a North American registry. JAMA Surg. 2018;153(9):826-833.

6. Koch CG, Li L, Duncan AI, et al. Morbidity and mortality risk associated with red blood cell and blood-component transfusion in isolated coronary artery bypass grafting. Crit Care Med. 2006;34(6):1608-1616.

7. Blanchette CM, Wang PF, Joshi AV, et al. Cost and utilization of blood transfusion associated with spinal surgeries in the United States. Eur Spine J. 2007;16(3):353-363.

8. Frank SM, Thakkar RN, Podlasek SJ, et al. Implementing a health system-wide patient blood management program with a clinical community approach. Anesthesiology. 2017;127(5):754-764.

9. Henry DA, Carless PA, Moxey AJ, et al. Anti-fibrinolytic use for minimising perioperative allogeneic blood transfusion. Cochrane Database Syst Rev. 2011;(3):CD001886.

10. Shander A, Hofmann A, Ozawa S, et al. Activity-based costs of blood transfusions in surgical patients at four hospitals. Transfusion. 2010;50(4):753-765.

11. Wang Q, Liu J, Fan R, et al. Tranexamic acid reduces postoperative blood loss of degenerative lumbar instability with 
stenosis in posterior approach lumbar surgery: a randomized controlled trial. Eur Spine J. 2013;22(9):2035-2038.

12. Neuman BJ, Ailon T, Scheer JK, et al. Development and validation of a novel adult spinal deformity surgical invasiveness score: analysis of 464 patients. Neurosurgery. 2018;82(6):847-853

13. Sethna NF, Zurakowski D, Brustowicz RM, et al. Tranexamic acid reduces intraoperative blood loss in pediatric patients undergoing scoliosis surgery. Anesthesiology. 2005;102(4):727-732.

14. Wong J, El Beheiry H, Rampersaud YR, et al. Tranexamic acid reduces perioperative blood loss in adult patients having spinal fusion surgery. Anesth Analg. 2008;107(5):1479-1486.

15. Elwatidy S, Jamjoom Z, Elgamal E, et al. Efficacy and safety of prophylactic large dose of tranexamic acid in spine surgery: a prospective, randomized, double-blind, placebo-controlled study. Spine (Phila Pa 1976). 2008;33(24):2577-2580.

16. Peters A, Verma K, Slobodyanyuk K, et al. Antifibrinolytics reduce blood loss in adult spinal deformity surgery: a prospective, randomized controlled trial. Spine (Phila Pa 1976). 2015;40(8):E443-E449.

17. Kushioka J, Yamashita T, Okuda S, et al. High-dose tranexamic acid reduces intraoperative and postoperative blood loss in posterior lumbar interbody fusion. J Neurosurg Spine. 2017;26(3):363-367.

18. McNicol ED, Tzortzopoulou A, Schumann R, et al. Antifibrinolytic agents for reducing blood loss in scoliosis surgery in children. Cochrane Database Syst Rev. 2016;9:CD006883.

19. Cheriyan T, Maier SP II, Bianco K, et al. Efficacy of tranexamic acid on surgical bleeding in spine surgery: a meta-analysis. Spine J. 2015;15(4):752-761.

20. Wang M, Zheng X-F, Jiang L-S. Efficacy and safety of antifibrinolytic agents in reducing perioperative blood loss and transfusion requirements in scoliosis surgery: a systematic review and meta-analysis. PLoS One. 2015;10(9):e0137886.

21. Yuan Q-M, Zhao Z-H, Xu B-S. Efficacy and safety of tranexamic acid in reducing blood loss in scoliosis surgery: a systematic review and meta-analysis. Eur Spine J. 2017;26(1):131-139.

22. Li G, Sun T-W, Luo G, Zhang C. Efficacy of antifibrinolytic agents on surgical bleeding and transfusion requirements in spine surgery: a meta-analysis. Eur Spine J. 2017;26(1):140154.

23. Zhong J, Cao K, Wang B, et al. The perioperative efficacy and safety of tranexamic acid in adolescent idiopathic scoliosis. World Neurosurg. 2019;129:e726-e732.

24. Xu D, Ren Z, Chen X, et al. The further exploration of hidden blood loss in posterior lumbar fusion surgery. Orthop Traumatol Surg Res. 2017;103(4):527-530.

25. Alshryda S, Mason J, Sarda P, et al. Topical (intra-articular) tranexamic acid reduces blood loss and transfusion rates following total hip replacement: a randomized controlled trial (TRANX-H). J Bone Joint Surg Am. 2013;95(21):1969-1974.

26. Demos HA, Lin ZX, Barfield WR, et al. Process improvement project using tranexamic acid is cost-effective in reducing blood loss and transfusions after total hip and total knee arthroplasty. J Arthroplasty. 2017;32(8):2375-2380.
27. National Clinical Guideline Centre. Cost-effectiveness analysis: tranexamic acid and cell salvage. In: Blood Transfusion. NICE Guideline No. 24. NICE, 2015

28. Wang D, Wang H-Y, Cao C, et al. Tranexamic acid in primary total knee arthroplasty without tourniquet: a randomized, controlled trial of oral versus intravenous versus topical administration. Sci Rep. 2018;8(1):13579.

29. Verma K, Errico T, Diefenbach C, et al. The relative efficacy of antifibrinolytics in adolescent idiopathic scoliosis: a prospective randomized trial. J Bone Joint Surg Am. 2014;96(10):e80.

30. Bradley KE, Ryan SP, Penrose CT, et al. Tranexamic acid or epsilon-aminocaproic acid in total joint arthroplasty? A randomized controlled trial. Bone Joint J. 2019;101-B(9):10931099.

31. Churchill JL, Puca KE, Meyer E, et al. Comparing $\varepsilon$-aminocaproic acid and tranexamic acid in reducing postoperative transfusions in total knee arthroplasty. J Knee Surg. 2017;30(5):460-466.

32. Churchill JL, Puca KE, Meyer ES, et al. Comparison of $\varepsilon$-aminocaproic acid and tranexamic acid in reducing postoperative transfusions in total hip arthroplasty. J Arthroplasty. 2016:31(12):2795-2799.e1.

33. Purvis TE, Goodwin CR, Molina CA, et al. Transfusion of red blood cells stored more than 28 days is associated with increased morbidity following spine surgery. Spine (Phila Pa 1976). 2018;43(13):947-953.

\section{Disclosures}

Ethan Cottrill reports receiving an F30 Grant from National Institutes of Health, National Institute on Aging (unrelated to the present study). Daniel M. Sciubba reports being a consultant for Baxter, DePuy-Synthes, Globus Medical, K2M, Medtronic, NuVasive, and Stryker and receiving grant funding from Baxter and Stryker.

\section{Author Contributions}

Conception and design: Sciubba, Ehresman, Pennington. Acquisition of data: Ehresman, Pennington, Schilling, Huq, Medikonda, Merkel, Ahmed, Lubelski, Farrokh, Frank. Analysis and interpretation of data: Sciubba, Ehresman, Pennington, Schilling, Huq, Ahmed, Westbroek, Frank. Drafting the article: Ehresman, Lubelski, Frank. Critically revising the article: Sciubba, Ehresman, Pennington, Medikonda, Merkel, Ahmed, Cottrill, Lubelski, Westbroek, Farrokh, Frank. Reviewed submitted version of manuscript: Sciubba, Ehresman, Pennington, Schilling, Huq, Medikonda, Merkel, Cottrill, Westbroek, Farrokh. Approved the final version of the manuscript on behalf of all authors: Sciubba. Statistical analysis: Ehresman.

\section{Correspondence}

Daniel M. Sciubba: Johns Hopkins School of Medicine, Baltimore,MD.dsciubb1@jhmi.edu. 